\title{
Micro/Nanobubbles Driven Multimodal Imaging and Theragnostics of Cancer
}

\author{
Xiaoting Zhang, BS ${ }^{a}$, Zhifei Dai, PhD ${ }^{a, *}$ \\ ${ }^{a}$ Department of Biomedical Engineering, College of Engineering, Peking University, Beijing, China \\ Received June 12, 2020; revision received June 29, 2020; accepted July 13, 2020
}

\begin{abstract}
Ultrasound imaging has attracted great interest of researchers due to their application in cancer diagnosis and treatment. Ultrasound contrast agents, microbubbles and nanobubbles are widely explored as a multifunctional platform, not only carrying other contrast agents for multimodal imaging to complement the disadvantages of each imaging modality, but also carrying drug/gene for cancer theragnostic. In this article, the characteristics and differences of microbubbles and nanobubbles are briefly introduced and reviewed. Besides, the microbubbles and nanobubbles driven multimodal imaging and theragnostic of cancer are summarized.
\end{abstract}

Key words: Microbubbles; Nanobubbles; Multimodal imaging; Theragnostic

Advanced Ultrasound in Diagnosis and Therapy 2021; 03: 163-172

DOI: $10.37015 /$ AUDT.2021.200053

$\mathrm{U}$ ltrasound (US) imaging is one of the most common non-invasive imaging methods in cancer diagnosis. Ultrasound imaging has the advantages of high safety, non-invasive nature, realtime imaging, deep penetration into tissue and low cost. In order to improve the accuracy and confidence of disease diagnosis, ultrasound contrast agents with high echogenicity are introduced to increase ultrasound signals and provide high-quality images. Gramiak et al. found a cloud of echoes in the heart chambers after intracardiac injection of indocyanine green, and first proposed the concept of ultrasound contrast agent in 1968 [1]. In 1972, Ziskin et al. found that the contrast effect could be produced by rapidly injection of any fluid, because of the production of bubble cavitation [2]. Since then, the study of bubbles as ultrasound contrast agents was developed rapidly [3, 4]. Microbubbles (MBs) are widely used as ultrasound contrast agents, usually composed of gaseous cores and outer shells of biocompatible materials, ranging from 1 to $10 \mu \mathrm{m}$ in diameter. Recently, nanobubbles (NBs) have been developed as novel ultrasound contrast agents $[5,6]$.

Although ultrasound imaging combined with contrast agents can provide higher resolution of tissues than that of ultrasound imaging only, there are still some limitations. Combination of ultrasound imaging with other imaging modalities can improve diagnostic accuracy and reliability, due to the unique advantages and limitations of each imaging modality. Microbubbles and nanobubbles are widely explored as a platform to carry other contrast agents for multimodal imaging [7-9].

Moreover, US plays a significant role in non-invasive therapy, such as high-intensity focused ultrasound (HIFU) $[10,11]$ and US-mediated drug delivery [12-14]. Focused ultrasound can generate thermal and non-thermal effect in target tissues, especially ultrasound-induced cavitation effects, which improve the drug delivery efficiency through increasing vascular permeability. The existence of microbubbles increases the delivery efficiency through enhancing ultrasound energy deposition and serving as cavitation nuclei [13]. Through binding drugs or gene to the shells or specific ligands, microbubbles and nanobubbles can serve as a multifunctional platform to deliver drugs or genes, increase local drug concentration, control drug release, and achieve simultaneously imaging and treatment of cancer.

\footnotetext{
* Corresponding author: Department of Biomedical Engineering, College of Engineering, Peking University, 5 Yiheyuan Road, Haidian District, Beijing, China.

e-mail: zhifei.dai@pku.edu.cn unrestricted use, distribution and reproduction in any medium provided that the original work is properly attributed.
} 
In this article, the characteristics and differences of microbubbles and nanobubbles are briefly introduced and reviewed. In addition, the microbubbles and nanobubbles driven multimodal imaging and theragnostic are summarized.

\section{Microbubbles and Nanobubbles}

Microbubbles are commonly used as ultrasound contrast agents in the clinic due to their excellent echogenicity. Several ultrasound contrast agents, such as Sonovue and Definity have been approved by the food and drug administration (FDA) for clinical applications. They can provide high quality ultrasound images of many organs and tissues, including heart, liver, spleen, blood vessels and lymph nodes. They also play a valuable role in tumor diagnosis and evaluation.

The physiochemistry of MBs are determined by the materials of gaseous cores and outer shells [15]. Air was used in first generation MBs, but abandoned because of poor stability. Then insoluble and low diffused gases, such as perfluorocarbons, are introduced to increases stability and the circulation time of microbubbles $[16,17]$. Biocompatible materials, including lipids, proteins [18], and polymers $[19,20]$ are primary choices for shell materials of MBs. The MBs can be functionalized by several methods [21-23], including attaching drugs to the surface of MBs, encasing hydrophobic drugs inside the microbubbles or embedding lipid-stabilized drugs within the microbubble membrane, as illustrated in Figure 1.

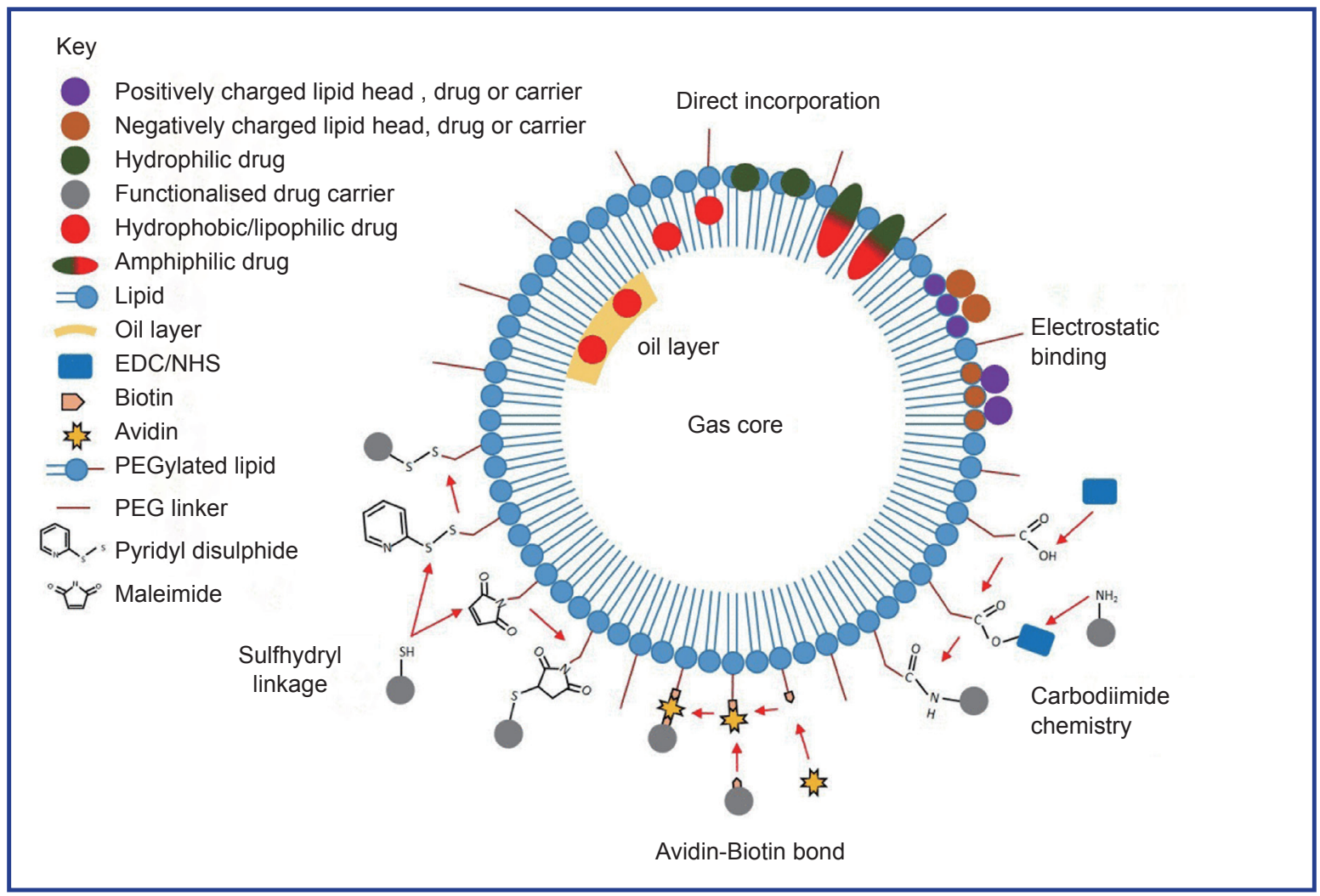

Figure 1 Schematic of different potential loading techniques for lipid microbubbles. All techniques are applicable to protein and polymer microbubbles [Reprinted with permission from reference 22].

Because the gaps between tumor vessels endothelium cells are smaller than $1 \mu \mathrm{m}$ [24], MBs cannot penetrate the tumor vessels and enter the tumor microenvironment. Nano-sized ultrasound contrast agents are small enough to penetrate tumor vessels pores and interact with tumor cells directly, thus showing great potential in the field of molecular ultrasound imaging and drug/gene delivery [25-27]. Nanobubbles have smaller particle size, more stable performance, and longer circulation time than MBs, which leads to more accumulation in the tumor area [28]. Researcher have confirmed that NBs can passively accumulate in the tumor area via the enhanced permeability and retention (EPR) effect [5]. Note that the echogenicity of NBs is decreased because of their small particle size, but it can be improved through shell modification and bubble aggregation [29].

Although MBs cannot penetrate tumor vessels and enter tumor tissues, ultrasound induced cavitation can enhance permeability of blood vessel wall and cell membrane, making it possible for MBs to deliver drug 
or genes into tumor microcirculation. The cavitation effect depends on various factors, including ultrasound frequency, intensity, microbubble shell composition and particle size [29]. Furthermore, under high acoustic pressures, microbubbles can violently contract and expand, even burst into smaller particles or fragments. Then carried drug/gene or obtained nanoparticles can be released and accumulated in tumor area via EPR effect. This approach is a safe and efficient active targeting method, which called ultrasound-targeted MB destruction (UTMD). The delivery induced by UTMD is limited to the area of ultrasound irradiation, thus it decreases the undesired damage to surrounding tissues and reduces systemic side effects. UTMD method is widely used in treatment of various types of cancer [30], cardiovascular diseases [31] and neurological diseases [32].

\section{Micro/Nanobubbles Mediated Multimodal Imaging}

Each imaging modality has its own advantages and limitations, thus multimodal imaging, which combines different imaging modalities, are developed to improve diagnostic accuracy and reliability. Microbubbles and nanobubbles are widely explored as a platform for multimodal imaging due to facile modification.

Fluorescence imaging has high sensitivity and possesses multicolor imaging capacity, while it suffers from low tissue penetration and poor spatial resolution. Considering these limitations, US/fluorescence bimodal imaging attracts great interest of researchers. With the introduction of fluorescence dyes, including indocyanine green (ICG) [33], cyanine dyes [34-37], quantum dot (QDs) [38,39] and porphyrin [40], microbubbles and nanobubbles can act as bimodal contrast agents for tumor visualization. Huynh et al. prepared porphyrin microbubbles (pMBs) as tri-modality contrast agents for ultrasound, fluorescence, and photoacoustic imaging [40]. Bacteriochlorophyll-lipid (BChl-lipid), a kind of porphyrin-lipid, was used as microbubble shell materials to provide fluorescence and photoacoustic properties. Upon exposure to low-frequency ultrasound, pMBs were successfully converted into porphyrin nanoparticles (pNPs), which generated only fluorescence and photoacoustic contrast signals. In vivo experiments also revealed the successful conversion of pMBs to pNPs and accumulation of pNPs at the tumor site without relying on the EPR effect. This conversion of MBs to NPs has great potential in imaging and therapeutic applications.

Targeted fluorescence nanobubbles plays a significant role in cancer diagnosis because of their small size and targeting character. $\mathrm{Li}$ et al. used biodegradable photoluminescent polymers (BPLPs) as a shell around liquid tetradecafluorohexane $\left(\mathrm{C}_{6} \mathrm{~F}_{14}\right)$ to form nanobubbles, followed by conjugation with PNBL-
NPY, a neuropeptide Y Y1 receptors (Y1R) ligand, for targeted breast tumor imaging [41]. In vitro and in vivo experiments demonstrated that the obtained Y1Rmediated fluorescent nanobubbles had good ultrasound contrast enhancement and targeting ability to Y1 receptors-overexpressing breast cancer. Near-infrared (NIR) heptamethine cyanine dyes with fluorescence property and tumor-targeting characteristic, such as IR-780 [42,43] and IR-783 [44], are loaded into the shell of nanobubbles for tumor-specific ultrasound/ fluorescence dual-modal imaging. For example, Shen et al. synthetized a novel targeted bimodal nanobubbles, FA-NBs-IR780, with a uniform nano-size $(591 \pm 52 \mathrm{~nm})$ [43]. Because folic acid (FA) and IR-780 iodide provided dual-targeting capacity, FA-NBs-IR780 could be efficiently accumulated at the tumor site for ultrasound/ fluorescence bimodal imaging. In addition, IR-780 iodide encapsulated in FA-NBs-IR780 can act as a photothermal agent to induce thermal ablation of tumors upon NIR irradiation with minimal damage to surrounding tissues. The nanobubbles combined with dual-targeting strategy have promising potentials for accurate diagnosis and treatment of cancer.

MRI provides worthwhile structural details of soft tissues with high spatial resolution, while its applications are limited by low sensitivity, long scanning time and high costs. MRI/ultrasound bimodal contrast agent has emerged as a promising method for cancer diagnosis. Superparamagnetic iron oxide (SPIO) nanoparticles are excellent T2-MRI contrast agents and can be physically embedded into the shell [45-47] or chemically conjugated on the external surface of MBs [48-50]. The echogenicity of the host MBs does not significantly decrease with the introduction of SPIO nanoparticles [49]. However, studies have demonstrated that the shell of MBs stiffen when SPIO entrapped into the shell. Thus, compared to MBs with SPIO embedded into the shell, MBs with external loading of SPIO have better performance for ultrasound imaging [51]. Chen et al. constructed novel microbubbles combined with manganese (III)-based MRI contrast agent for bimodal tumor imaging [52]. Mnchelated porphyrin lipid (MnP) were fabricated to form the shell of microbubbles, followed by encapsulating a perfluoropropane gas core. The Mn-chelated porphyrin microbubbles (MnP-MBs) possessed good contrast enhancement ability for both US and MR imaging with a very low dose. Upon low-intensity US exposure, MnPMBs were successfully converted into NPs and exhibited high accumulation at the tumor site for efficient MRI contrast enhancement (Fig. 2).

The combination of three-dimensional $\mathrm{CT}$ and realtime ultrasound provides accurate images to identify the size and location of tumors, as well as to monitor 
and guide cancer therapy. US/CT bimodal contrast agents were successfully fabricated by introduction of gold nanoparticles (GNP) into microbubbles [53] and nanobubbles [54]. Ke et al. fabricated gold nanoshelled perfluorooctylbromide (PFOB) nanocapsules with PEGylation (PGsP NCs) for ultrasound/CT dualmodal contrast imaging [54]. The gold nanoshell on the surface provided CT signals, and encapsulated PFOB of PGsP NCs provided ultrasound contrast signals. Moreover, gold nanoshell could induce thermal ablation of tumors upon laser irradiation due to the excellent photothermal property of gold nanoparticles. The use of multifunctional nanobubbles offers accurate diagnostic information to improve imaging-guided cancer treatment. Other CT contrast agents, such as iodinate compounds [55] and bismuth sulfide $\left(\mathrm{Bi}_{2} \mathrm{~S}_{3}\right)$ [56], are also used to constructed dual-modal contrast agents for more accurate and comprehensive diagnosis of tumor. Zhou et al. constructed folate-targeted perfluorohexane nanoparticles carrying bismuth sulfide (FLBS-PFH-NPs) for tumor ultrasound/CT imaging and targeted HIFU therapy [56]. Upon ultrasonic irradiation, the capsulated $\mathrm{PFH}$ underwent a liquid-gas phase-transition, then the formation of MBs enhanced the efficacy of HIFU therapy. The as-designed multifunctional contrast agents depending on active targeting significantly improved the diagnosis and treatment effect of cancer.
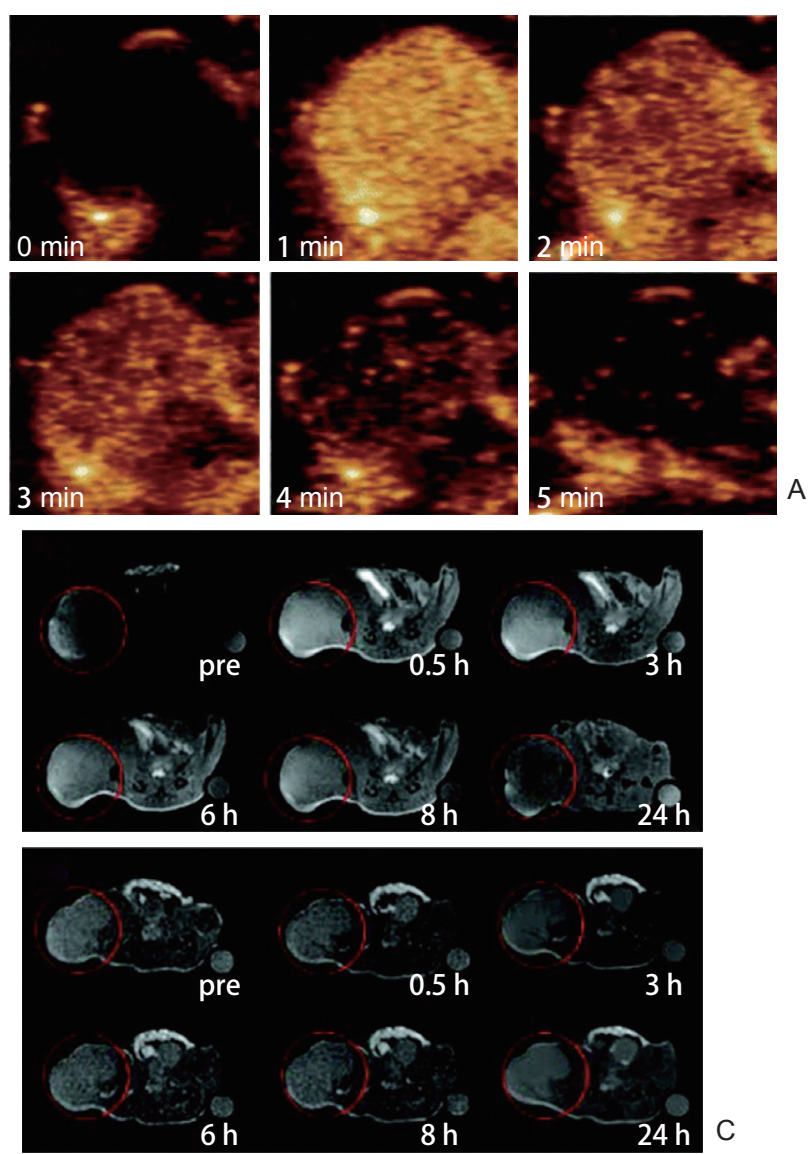
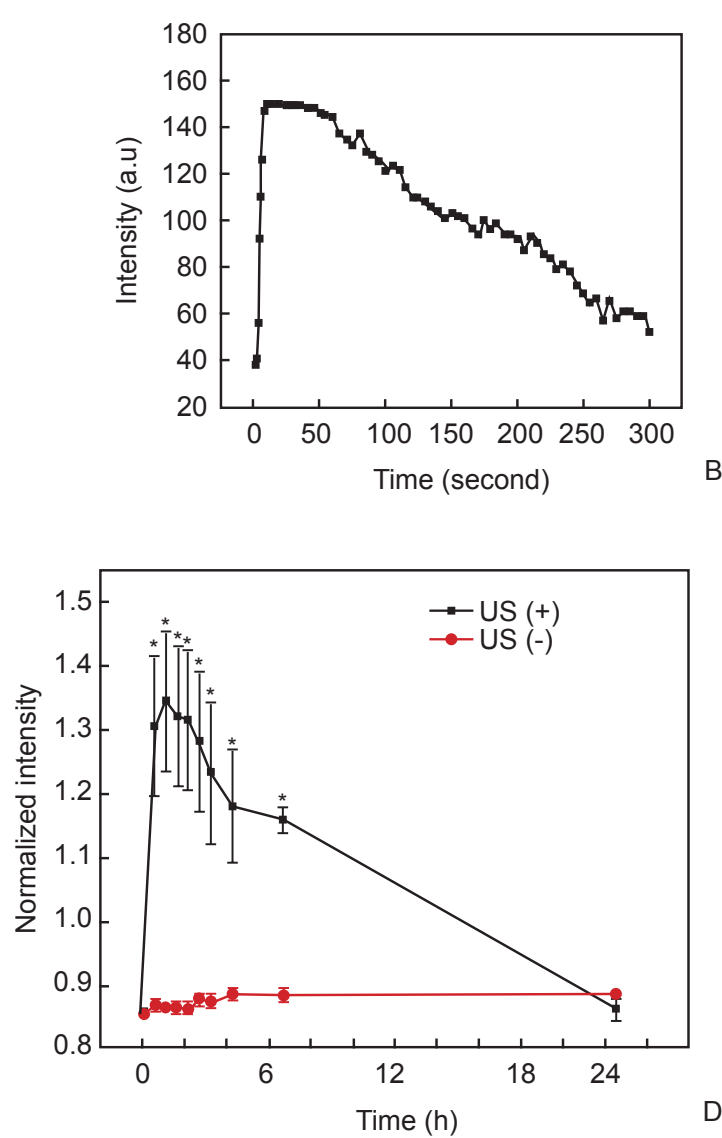

Figure 2 (A-B) In vivo US imaging of MnP-MBs. MnP-MBs were intravenously administered into U87 glioblastoma tumor-bearing nude mice. The duration of US contrast enhancement in the tumor was monitored by a clinical US probe and the quantitative data are shown in (B); (C-D) In vivo MRI imaging of MnP-MBs. In vivo MRI monitoring of MnP-MBs with US exposure (C, upper) and without US exposure (C, bottom) and their quantitative results (D) within $24 \mathrm{~h}$. [Reprinted with permission from reference 52]

\section{Micro/Nanobubbles Driven Theragnostics of Cancer}

With the guidance of ultrasound, microbubbles enable not only ultrasound contrast imaging for diagnosis, but also increasing vascular permeability and promoting drug/gene penetration into tumors. It is feasible to deliver drugs or genes through co-injection of microbubbles with drugs followed by MBs induced sonoporation [57-59]. For example, the combination of gemcitabine, SonoVue ${ }^{\circledR}$ and ultrasound has been applied for the treatment of pancreatic ductal adenocarcinoma [57]. The results of Phase I clinical trial proved that the combined therapeutic regimen enhanced the treatment effect, improved life quality of patients and prolonged their survival without additional side effects. However, incorporating drugs into MBs is a better choice, which ensures the same bio-distribution and pharmacodynamic behaviors of drugs and MBs. 


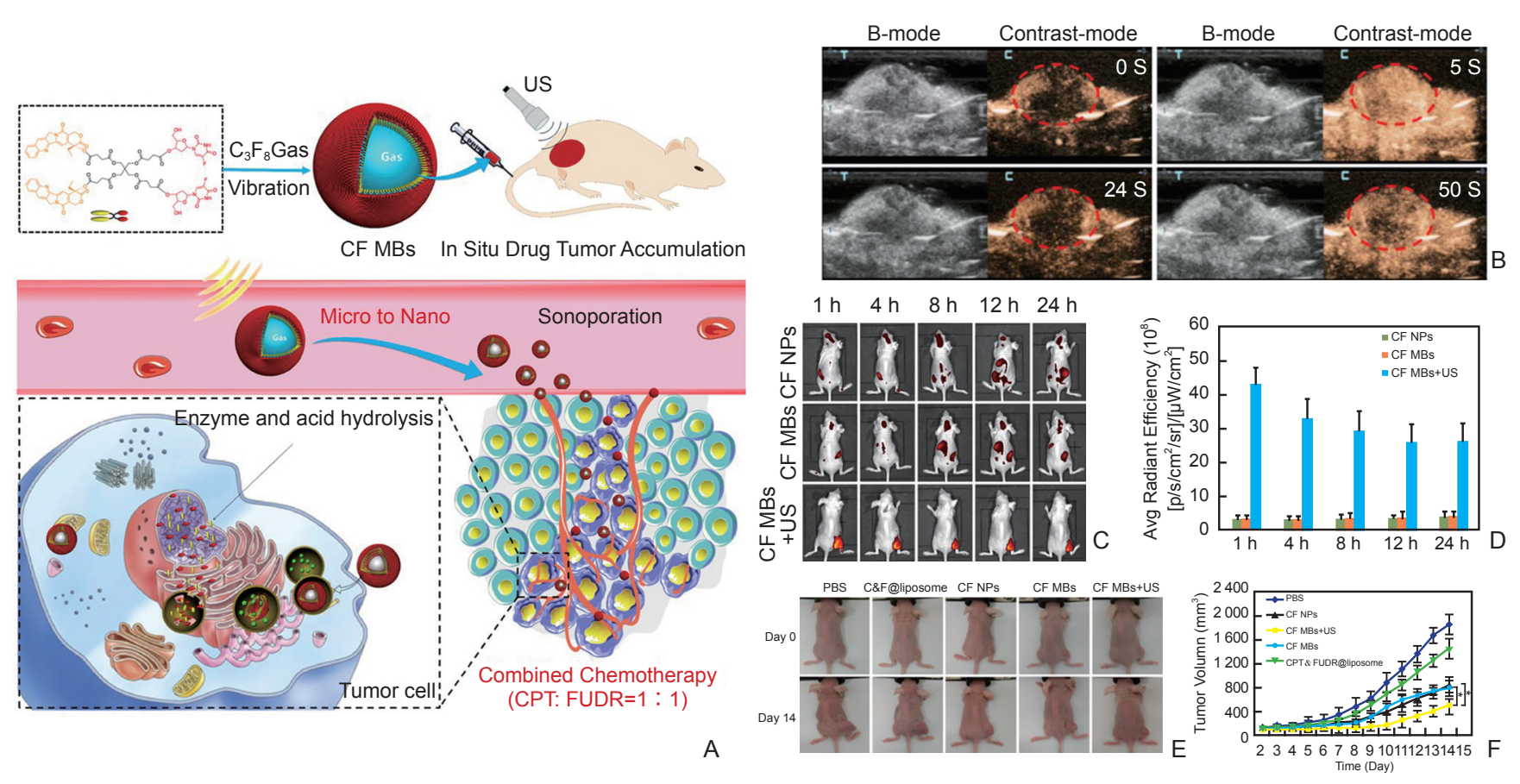

Figure 3 (A) Schematic illustration of engineering and US-induced micro-to-nano conversion of Janus camptothecin-floxuridine MBs (CF MBs) for in situ combined cancer chemotherapy; (B) In vivo US imaging. CF MBs were intravenously administered into 4T1 tumor-bearing mice, and the tumor was imaged with a clinical US probe (3 12 MHz); (C) Time-lapse NIR fluorescence imaging of mice; (D) Quantitation of tumor site after tail vein injection. (EF) In vivo antitumor effect of CF MBs combined with US on 4T1 tumor-bearing nude mice; (E) Images of tumor-bearing mice after different treatments; (F) Tumor volume change over time after treatment with CPT\&FUDR@liposome, CF NPs, CF MBs and CF MBs + US, ${ }^{*} P<0.05$. [Reprinted with permission from reference 71]

\section{Multifunctional Micro/Nanobubbles Driven}

\section{Chemotherapy}

Chemotherapy plays a significant role in cancer treatment, but it faces with many problems, including low bioavailability, side effects and drug-resistance. Drug-loaded MBs combined with ultrasound provide an effective strategy to increase local drug concentration, control drug release, and reduce side effects and drugresistance. Common chemotherapy drugs, such as doxorubicin [60-63], docetaxel [64,65], and paclitaxel $[66,67]$ have been loaded into MBs for application of cancer theragnostics. Ting et al. prepared MBs loaded with 1,3-bis(2-chloroethyl)-1-nitrosourea (BCNU) for brain tumor treatment [68]. The blood-brain barrier (BBB) protects the brain from potentially harmful compounds in the blood, but it restricts the chemotherapy drugs entering brain tumors. MBs-mediated focused ultrasound (FUS) provides a feasible strategy to induce local BBB opening and improve drug delivery into brain. The combination of FUS (1MHz) with BCNU-MBs successfully triggered BBB opening, meanwhile BCNU was released from the BCNU-MBs and accumulated at the target site, thereby significantly inhibiting glioma growth and prolonging survival of tumor-bearing rats. Furthermore, multifunctional MBs loaded with DOX and SPIO nanoparticles were designed by the same group
$[60,69]$. The present of SPIO particles not only acted as T2-MRI contrast agents, but also allowed magnetic targeting (MT) to enhance drug delivery to brain tumors. The designed multifunctional MBs loaded with DOX and conjugated with SPIO nanoparticles (DOX-SPIO-MBs) exhibited good contrast enhancement in both US and MR imaging [69]. The DOX-SPIO-MBs simultaneously induced BBB opening and drug delivery with FUS exposure, then magnetic targeting (MT) further enhanced drug accumulation in brain tumors. They also proposed a novel theragnostic complex of SPIO-DOX-conjugated MB (SD-MBs) for drug delivery via ultrasound/magnetic dual-targeting [60]. The behaviors of DOX could be real-time monitored by MRI signals of SD complex. Such dual-targeting therapeutic strategy has promising potentials to achieve advanced tumor treatment.

Through further conjugation with targeting ligands, the chemotherapy MBs and NBs can be used as potential tumor-targeted theragnostic agents for future applications. Zhang et al. fabricated iRGD-modified paclitaxel-loaded liposomes (iRGD-PTX-PL), then successfully conjugated it to the surface of MBs through biotin-avidin linkage [67]. The obtained liposome-microbubble complexes (iRGD-PTX-LMC) had high affinity for breast tumors and great ultrasonic responsiveness for local drug release upon ultrasound irradiation. Compared with iRGDPTX-PL and nontargeted PTX-LMC, the combination of 
iRGD-PTX-LMC and ultrasound could better improve local drug delivery and enhance therapeutic effect. Gao et al. designed folic acid (FA)-conjugated lipid nanobubbles loading artesunate (Arte; FA-ALNBs) for ultrasound-guided targeted chemotherapy [70]. The FAALNBs had a uniform particle size distribution (781.2 \pm $5.3 \mathrm{~nm}$ ), good stability in physiological environment and excellent capability to enhance US signals. Upon FUS irradiation, the entrapped Arte was released as a result of US-mediated FA-ALNBs destruction. The FA receptor-mediated endocytosis process enhanced the cellular uptake of FA-ALNBs. Both in vitro and in vivo experiments demonstrated that FA-ALNBs combined with US exhibited great anti-tumor effect without obvious systemic toxicity.

Recent studies have revealed that one of major limitations of MB-driven chemotherapy is their low drug loading content. To enhance the chemotherapeutic efficacy with minimal side effects, Liang et al. synthesized an amphiphilic Janus camptothecinfloxuridine (CPT-FUDR, CF) conjugate as shell materials to construct $\mathrm{CF}$ MBs with high drug loading contents $(\sim 56.7 \%)$ [71]. CF MBs showed excellent US contrast capability. Upon UTMD technique with the guidance of ultrasound imaging, CF MBs were converted into nanoparticles in situ, resulting in $\sim 14$ times higher drug accumulation in subcutaneous breast tumor and reduced undesired drug accumulation in organs. Furthermore, the ester bond of $\mathrm{CF}$ was hydrolyzed in the tumor microenvironment, and CPT and FUDR were released at an exact 1:1 ratio to achieved synergistic chemotherapy for tumors. The tumor growth inhibition ratio of CF MBs combined with US was $72.4 \%$, higher than that of CF NPs $(54.1 \%)$ and liposomes loaded with CPT and FUDR (21.6\%), which demonstrated that CF MBs combined with UTMD is an effective strategy for cancer theragnostics (Fig. 3). Based on CF MBs, Chen et al. successfully constructed porphyrin/camptothecinfloxuridine triad MBs (PCF-MBs) with high drug loading contents for ultrasound/fluorescence bimodal imaging and chemo-photodynamic combination therapy [72]. PCF-MBs induced PDT could reduce the expression of ATP-binding cassette subfamily G member 2 (ABCG2), thus overcoming multidrug resistance (MDR) in colorectal cancer. This combination therapeutic strategy based on PCF-MBs demonstrated a 90\% tumor inhibition ratio in murine colon cancer models without recurrence.

\section{Multifunctional micro/nanobubbles driven gene therapy}

Gene delivery using ultrasound and microbubbles is another attractive approach for tumor treatment. The cationic microbubbles are suitable to deliver gene via charge-coupling with genetic material such as DNA [73,
74], microRNA (miRNA) [75] and small interfering RNA (siRNA) [76]. UTMD effectively improves gene transfection efficiency with the advantages of safety, practicality, and target. The use of miRNA-133amicrobubbles with UTMD for breast cancer therapy was reported by $\mathrm{Ji}$ et al. [75]. When miR-133a-microbubble injected into animal model of breast cancer, miR-133a presented prolonged circulation time, and showed better delivery efficiency upon low-frequency ultrasound. UTMD of miR-133a was a promising approach to suppressing the tumor growth and improving survival of xenografted mouse model. Zhou et al. developed targeted cationic microbubbles conjugated with CD105 antibody (CMB105), following by loading endostatin gene to achieve targeted anti-angiogenesis gene therapy and ultrasound imaging [73]. The CD105 antibody conjugated on the surface of CMB105 would not affect the binding ability of plasmid DNA. Compared with untargeted cationic microbubbles (CMB) and neutral microbubbles (NMB), CMB105 combined with UTMD presented better gene transfection efficiency and treatment effect in vivo.

Nanobubbles are potential multifunctional carriers for gene delivery [77]. Nanobubbles could pass through tumor blood vessels and passively accumulate in tumor microcirculation due to their nanoscale size. Cai et al. successfully synthesized NBs carrying siRNA (NBssiRNA) by a biotin-streptavidin system [76]. The NBssiRNA had high ultrasound contrast-enhancement capability and can be disrupted to induce sonoporation when exposure to ultrasound. The NBs-siRNA combined with ultrasound targeted destruction (UTD) possessed better transfection efficiency of siRNA than siRNA-NBs without UTD. In vivo experiment demonstrated siRNANBs combined with UTD could successfully deliver siRNA into tumor tissues to inhibit glioma tumor growth and prolong survival of mice.

\section{Multifunctional micro/nanobubbles driven synergistic therapy}

There are also theragnostic MBs and NBs with sophisticated designs that combine photothermal therapy [78-80], photodynamic therapy [81], and synergistic therapy $[72,82,83]$. For example, NIR fluorescence dye 1,1-dioctadecyl-3, 3, 3, 3-tetramethylindotricarbocya nine iodide (DiR) can act as a photothermal agent for tumor photoablation, while porphyrin and chlorin e6 (Ce6) can operate as a photosensitizer for photodynamic therapy. $\mathrm{Xu}$ et al. prepared multifunctional theragnostic microbubbles by self-assembly from porphyrin grafted lipid (PGL) and DiR with high loading contents (PGL: 5.8\%; DiR: 10.38\%) [84]. The PGL-DiR MBs showed excellent ultrasound/fluorescence dual-modal contrast 
capability, and could be used for combined photothermal therapy and photodynamic therapy with the assistance of UTMD. Upon US exposure to tumor, the PGL-DiR MBs were converted into nanoparticles, resulting in improved accumulation and cellular internalization of two phototherapeutic agents. With the laser irradiation for PDT and PTT, the tumor growth of xenografted mouse model treated with PGL-DiR MBs were completely inhibited with no recurrence, while the tumor growth inhibition ratio of PGL-DiR NPs was only $72.6 \%$ (Fig. 4 ). The combination of phototherapy with PGL-DiR MBs provided an effective strategy for imaging-guided tumor therapy. However, previous studies suggest that PDT may induce undesired overexpression of angiogenic factors in tumor [85]. To overcome this side effect, Jang et al. developed the complex of DOX-encapsulating antiangiogenic siRNA nanoparticle and Ce6-encapsulating microbubble (DOX-siVEGF-NPs/Ce6-MBs) for combination of chemotherapy, photodynamic and gene therapy [83]. With local ultrasound irradiation, the DOXsiVEGF-NPs/Ce6-MBs was disrupted and released three drugs at the tumor microenvironment, resulted in effective inhibition of angiogenesis and enhanced therapeutic outcomes.
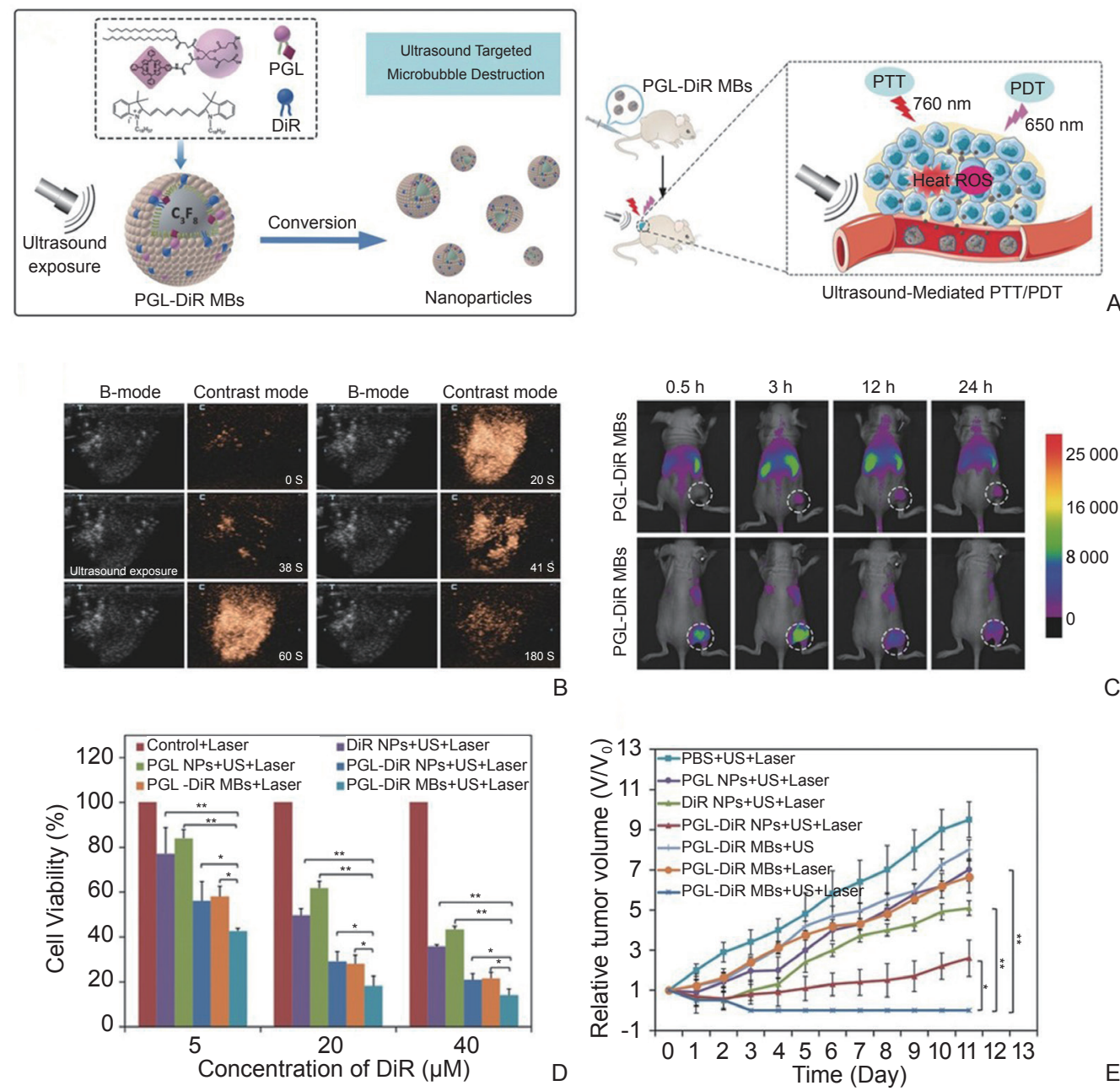

C

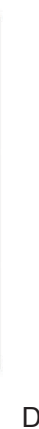

$\mathrm{E}$

Figure 4 (A) The structure and conversion of the PGL-DiR MBs into nanoparticles when exposed to sufficient ultrasound, and ultrasound-mediated tumorspecific combined photothermal and photodynamic therapy; (B) PGL-DiR MBs were intravenously administrated into the 4T1 tumor-bearing mice and the tumor was imaged with a clinical ultrasound probe (3-12 MHz). At $38 \mathrm{~s}$, the tumor site was irradiated by the low-frequency ultrasound with a higher mechanical index to destroy the MBs; (C) Time-lapse NIR fluorescence imaging of mice intravenously administrated with PGL-DiR NPs, and PGL-DiR MBs combined with ultrasound exposure. The dashed circles indicate subcutaneous tumor regions; (D) Cell viabilities of 4T1 cells treated by PGL NPs+US, DiR NPs+US, PGL-DiR NPs+US, PGL-DiR MBs, and PGL-DiR MBs+US. All the above groups were irradiated by $760 \mathrm{~nm}$ laser $\left(1 \mathrm{~W} \mathrm{~cm}{ }^{-2}, 10 \mathrm{~min}\right)$ and $650 \mathrm{~nm}$ laser (200 mW cm $\mathrm{m}^{-2}, 3 \mathrm{~min}$ ). The molar concentrations of PGL and DiR were same; (E) Tumor growth curves of different groups of mice received various treatments indicated. The data were presented as the mean $\pm \mathrm{SD}(n=5),{ }^{*} P<0.05,{ }^{* *} P<0.01$. [Reprinted with permission from reference 84]

\section{Conclusion}

Ultrasound imaging is an important diagnostic tool in the clinical settings due to the advantages of high safety, non-invasive nature, real-time imaging, deep penetration into tissue and cost effectiveness. Microbubbles and nanobubbles are widely used as ultrasound contrast agents for tumor diagnostics. Several microbubbles have been approved by FDA for diagnostic applications. 
Multimodal imaging can be achieved through loading certain contrast agents in microbubbles and nanobubbles. Similarly, microbubbles and nanobubbles play a significant role to deliver drug/gene for tumor treatment.

However, multifunctional MBs and NBs still suffer from limitations that confine the clinical application to some extent. One of major limitations is low delivery efficacy due to instability and short circulation time of MBs and NBs. We need to consider the choice of membrane material, size distributions and ultrasonic parameters to overcome this for effective delivery into tumors. UTMD technique is a useful targeting approach to improve drug delivery and local release. To get better therapeutic effect, it is necessary to improve drug loading of MBs and NBs. Researchers have developed novel shell materials composed of drugs. Conjugating drug liposomes to the surface of microbubbles also increase drug loading for chemotherapy and other related applications. In addition, the bioeffects associated with multifunctional MBs and NBs, including toxicity, biodistribution and pharmacokinetics should be investigated as clinical translation proceeds. Believing that with further research on multifunctional MBs and NBs, it has a wide application prospect in the clinical practice.

\section{Acknowledgements}

This contribution was financially supported by National Key Research and Development Program of China (No. 2016YFA0201400), State Key Program of National Natural Science of China (No. 81930047), Projects of International Cooperation and Exchanges NSFC-PSF (No. 31961143003), National Project for Research and Development of Major Scientific Instruments (No. 81727803), Beijing Natural Science Foundation, Haidian, Original Innovation Joint Fund (No. 17L20170), and the Foundation for Innovative Research Groups of the National Natural Science Foundation of China (No. 81421004).

\section{Conflict of Interest}

The authors have declared no conflicts of interest for this article.

\section{References}

[1] Gramiak R, Shah PM. Echocardiography of the aortic root. Invest Radiol 1968; 3: 356-366.

[2] Ziskin MC, Bonakdarpour A, Weinstein DP, Lynch PR. Contrast agents for diagnostic ultrasound. Invest Radiol 1972; 7: 500-505.

[3] Lindner JR. Microbubbles in medical imaging: current applications and future directions. Nat Rev Drug Discov 2004; 3: 527-532.

[4] Quaia E. Microbubble ultrasound contrast agents: an update. Eur Radiol 2007; 17: 1995-2008.

[5] Yin T, Wang P, Zheng R, Zheng B, Cheng D, Zhang X, et al. Nanobubbles for enhanced ultrasound imaging of tumors. Int $J$ Nanomedicine 2012;7:895-904.

[6] Wheatley MA, Forsberg F, Dube N, Patel M, Oeffinger BE.
Surfactant-stabilized contrast agent on the nanoscale for diagnostic ultrasound imaging. Ultrasound Med Biol 2006; 32: 83-93.

[7] Lin Y, Chen ZY, Yang F. Ultrasound-based multimodal molecular imaging and functional ultrasound contrast agents. Curr Pharm Des 2013; 19: 3342-3351.

[8] Li Y, Chen Y, Du M, Chen ZY. Ultrasound technology for molecular imaging: from contrast agents to multimodal imaging. ACS Biomater Sci Eng 2018; 4: 2716-2728.

[9] Xing Z, Wang J, Ke H, Zhao B, Yue X, Dai Z, et al. The fabrication of novel nanobubble ultrasound contrast agent for potential tumor imaging. Nanotechnology 2010; 21: 145607.

[10] Poissonnier L, Chapelon JY, Rouvière O, Curiel L, Bouvier R, Martin X, et al. Control of prostate cancer by transrectal HIFU in 227 patients. Eur Urol 2007; 51: 381-387.

[11] Zhang L, Zhu H, Jin C, Zhou K, Li K, Su H, et al. High-intensity focused ultrasound (HIFU): effective and safe therapy for hepatocellular carcinoma adjacent to major hepatic veins. Eur Radiol 2009; 19: 437-445.

[12] Unger E, Porter T, Lindner J, Grayburn P. Cardiovascular drug delivery with ultrasound and microbubbles. Adv Drug Deliv Rev 2014; 72: 110-126.

[13] Hernot S, Klibanov AL. Microbubbles in ultrasound-triggered drug and gene delivery. Adv Drug Deliv Rev 2008; 60: 1153-1166.

[14] Dasgupta A, Liu M, Ojha T, Storm G, Kiessling F, Lammers T. Ultrasound-mediated drug delivery to the brain: principles, progress and prospects. Drug Discov Today Technol 2016; 20: 41-48.

[15] Ferrara K, Pollard R, Borden M. Ultrasound microbubble contrast agents: fundamentals and application to gene and drug delivery. Annu Rev Biomed Eng 2007; 9: 415-447.

[16] Schutt EG, Klein DH, Mattrey RM, Riess JG. Injectable microbubbles as contrast agents for diagnostic ultrasound imaging: the key role of perfluorochemicals. Angew Chem Int Ed Engl 2003; 42: 3218-3235.

[17] Kabalnov A, Bradley J, Flaim S, Klein D, Pelura T, Peters B, et al Dissolution of multicomponent microbubbles in the bloodstream: 2 . Experiment. Ultrasound Med Biol 1998; 24: 751-760.

[18] Feinstein SB, Cheirif J, Ten Cate FJ, Silverman PR, Heidenreich PA, Dick C, et al. Safety and efficacy of a new transpulmonary ultrasound contrast agent: initial multicenter clinical results. J Am Coll Cardiol 1990; 16: 316-324.

[19] Wheatley MA, Schrope B, Shen P. Contrast agents for diagnostic ultrasound: development and evaluation of polymer-coated microbubbles. Biomaterials 1990; 11: 713-717.

[20] Xing Z, Ke H, Wang J, Zhao B, Yue X, Dai Z, et al. Novel ultrasound contrast agent based on microbubbles generated from surfactant mixtures of Span 60 and polyoxyethylene 40 stearate. Acta Biomater 2010; 6: 3542-3549.

[21] Gong Q, Gao X, Liu W, Hong T, Chen C. Drug-loaded microbubbles combined with ultrasound for thrombolysis and malignant tumor therapy. Biomed Res Int 2019; 2019: 6792465.

[22] Mulvana H, Browning RJ, Luan Y, de Jong N, Tang MX, Eckersley RJ, et al. Characterization of contrast agent microbubbles for ultrasound imaging and therapy research. IEEE Trans Ultrason Ferroelectr Freq Control 2017; 64: 232-251.

[23] Fu L, Ke HT. Nanomaterials incorporated ultrasound contrast agents for cancer theranostics. Cancer Biol Med 2016; 13: 313-324.

[24] Hobbs SK, Monsky WL, Yuan F, Roberts WG, Griffith L, Torchilin VP, et al. Regulation of transport pathways in tumor vessels: role of tumor type and microenvironment. Proc Natl Acad Sci U S A 1998; 95: 4607-4612.

[25] Perera RH, Hernandez C, Zhou H, Kota P, Burke A, Exner AA. Ultrasound imaging beyond the vasculature with new generation 
contrast agents. Wiley Interdiscip Rev Nanomed Nanobiotechnol 2015; 7: 593-608

[26] Zullino S, Argenziano M, Stura I, Guiot C, Cavalli R. From microto nano-multifunctional theranostic platform: effective ultrasound imaging is not just a matter of scale. Mol Imaging 2018; 17: 1536012118778216.

[27] Yang H, Cai W, Xu L, Lv X, Qiao Y, Li P, et al. Nanobubbleaffibody: novel ultrasound contrast agents for targeted molecular ultrasound imaging of tumor. Biomaterials 2015; 37: 279-288.

[28] Wu H, Abenojar EC, Perera R, De Leon AC, An T, Exner AA. Timeintensity-curve analysis and tumor extravasation of nanobubble ultrasound contrast agents. Ultrasound Med Biol 2019; 45: 25022514

[29] Duan L, Yang L, Jin J, Yang F, Liu D, Hu K, et al. Micro/nanobubble-assisted ultrasound to enhance the EPR effect and potential theranostic applications. Theranostics 2020; 10: 462-483.

[30] Hameed S, Zhang M, Bhattarai P, Mustafa G, Dai Z. Enhancing cancer therapeutic efficacy through ultrasound-mediated micro-tonano conversion. Wiley Interdiscip Rev Nanomed Nanobiotechnol 2020; 12: e1604.

[31] Deng Q, Hu B, Cao S, Song HN, Chen JL, Zhou Q. Improving the efficacy of therapeutic angiogenesis by UTMD-mediated Ang-1 gene delivery to the infarcted myocardium. Int J Mol Med 2015; 36: 335344.

[32] Zhang N, Yan F, Liang X, Wu M, Shen Y, Chen M, et al. Localized delivery of curcumin into brain with polysorbate 80 -modified cerasomes by ultrasound-targeted microbubble destruction for improved Parkinson's disease therapy. Theranostics 2018; 8: 22642277.

[33] Xu RX, Huang J, Xu JS, Sun D, Hinkle GH, Martin EW, et al. Fabrication of indocyanine green encapsulated biodegradable microbubbles for structural and functional imaging of cancer. $J$ Biomed Opt 2009; 14: 034020.

[34] Zhang X, Liu R, Dai Z. Multicolor nanobubbles for FRET/ultrasound dual-modal contrast imaging. Nanoscale 2018; 10: 20347-20353.

[35] Lin X, Zhang X, Wang S, Liang X, Xu Y, Chen M, et al. Intraoperative identification and guidance of breast cancer microfoci using ultrasound and near-infrared fluorescence dual-modality imaging. ACS Appl Bio Mater 2019;2(5):2252-2261.

[36] Liu R, Tang J, Xu Y, Dai Z. Bioluminescence imaging of inflammation in vivo based on bioluminescence and fluorescence resonance energy transfer using nanobubble ultrasound contrast agent. ACS Nano 2019; 13: 5124-5132.

[37] Qu E, Dai Z, Liang X, Qian Y, Wang S, Ke H, et al. Detection and pathologic evaluation of sentinel lymph nodes in the vx2 tumor model using a novel ultrasound/near-infrared dual-modality contrast agent. Ultrasound Med Biol 2015; 41: 1905-1912.

[38] Ke H, Xing Z, Zhao B, Wang J, Liu J, Guo C, et al. Quantumdot-modified microbubbles with bi-mode imaging capabilities. Nanotechnology 2009; 20: 425105.

[39] Yang Y, Wang J, Li X, Lin L, Yue X. A near infrared fluorescent/ ultrasonic bimodal contrast agent for imaging guided pDNA delivery via ultrasound targeted microbubble destruction. RSC $A d v$ 2015;5(11):8404-8414.

[40] Huynh E, Leung BY, Helfield BL, Shakiba M, Gandier JA, Jin CS, et al. In situ conversion of porphyrin microbubbles to nanoparticles for multimodality imaging. Nat Nanotechnol 2015; 10: 325-332.

[41] Li J, Tian Y, Shan D, Gong A, Zeng L, Ren W, et al. Neuropeptide Y Y1 receptor-mediated biodegradable photoluminescent nanobubbles as ultrasound contrast agents for targeted breast cancer imaging. Biomaterials 2017; 116: 106-117.

[42] Yang H, Zhou T, Cai W, Yi X, Liu X, Wang Y, et al. Novel dual-mode nanobubbles as potential targeted contrast agents for female tumors exploration. Tumour Biol 2016; 37: 14153-14163.

[43] Shen Y, Lv W, Yang H, Cai W, Zhao P, Zhang L, et al. FA-NBsIR780: Novel multifunctional nanobubbles as molecule-targeted ultrasound contrast agents for accurate diagnosis and photothermal therapy of cancer. Cancer Lett 2019; 455: 14-25.

[44] Lv W, Shen Y, Yang H, Yang R, Cai W, Zhang J, et al. A novel bimodal imaging agent targeting HER2 molecule of breast cancer. $J$ Immunol Res 2018; 2018: 6202876

[45] Liu Z, Lammers T, Ehling J, Fokong S, Bornemann J, Kiessling F, et al. Iron oxide nanoparticle-containing microbubble composites as contrast agents for MR and ultrasound dual-modality imaging. Biomaterials 2011; 32: 6155-6163.

[46] Sun Y, Zhu Y, Huang C, Li R, Chen Y, Duan Y. Magnetite loaded Polypeptide-PLGA multifunctional microbubbles for dual-mode US/ MR imaging. Contrast Media Mol Imaging 2016; 11: 146-153.

[47] Yang F, Li Y, Chen Z, Zhang Y, Wu J, Gu N. Superparamagnetic iron oxide nanoparticle-embedded encapsulated microbubbles as dual contrast agents of magnetic resonance and ultrasound imaging. Biomaterials 2009; 30: 3882-3890.

[48] Duan L, Yang F, Song L, Fang K, Tian J, Liang Y, et al. Controlled assembly of magnetic nanoparticles on microbubbles for multimodal imaging. Soft Matter 2015; 11: 5492-5500.

[49] Brismar TB, Grishenkov D, Gustafsson B, Härmark J, Barrefelt A, Kothapalli SV, et al. Magnetite nanoparticles can be coupled to microbubbles to support multimodal imaging. Biomacromolecules 2012; 13: 1390-1399.

[50] Beguin E, Bau L, Shrivastava S, Stride E. Comparing strategies for magnetic functionalization of microbubbles. ACS Appl Mater Interfaces 2019; 11: 1829-1840.

[51] Sciallero C, Balbi L, Paradossi G, Trucco A. Magnetic resonance and ultrasound contrast imaging of polymer-shelled microbubbles loaded with iron oxide nanoparticles. $R$ Soc Open Sci 2016; 3: 160063.

[52] Chen M, Liang X, Dai Z. Manganese(iii)-chelated porphyrin microbubbles for enhanced ultrasound/MR bimodal tumor imaging through ultrasound-mediated micro-to-nano conversion. Nanoscale 2019; 11: 10178-10182.

[53] Jin Y, Wang J, Ke H, Wang S, Dai Z. Graphene oxide modified PLA microcapsules containing gold nanoparticles for ultrasonic/CT bimodal imaging guided photothermal tumor therapy. Biomaterials 2013; 34: 4794-4802.

[54] Ke H, Yue X, Wang J, Xing S, Zhang Q, Dai Z, et al. Gold nanoshelled liquid perfluorocarbon nanocapsules for combined dual modal ultrasound/CT imaging and photothermal therapy of cancer. Small 2014; 10: 1220-1227.

[55] Choi D, Jeon S, You DG, Um W, Kim JY, Yoon HY, et al. Iodinated echogenic glycol chitosan nanoparticles for X-ray CT/US dual imaging of tumor. Nanotheranostics 2018; 2: 117-127.

[56] Zhou D, Li C, He M, Ma M, Li P, Gong Y, et al. Folate-targeted perfluorohexane nanoparticles carrying bismuth sulfide for use in US/CT dual-mode imaging and synergistic high-intensity focused ultrasound ablation of cervical cancer. J Mater Chem B 2016; 4: 4164-4181.

[57] Dimcevski G, Kotopoulis S, Bjånes T, Hoem D, Schjøtt J, Gjertsen $\mathrm{BT}$, et al. A human clinical trial using ultrasound and microbubbles to enhance gemcitabine treatment of inoperable pancreatic cancer. $J$ Control Release 2016; 243: 172-181.

[58] Du J, Sun Y, Li FH, Du LF, Duan YR. Enhanced delivery of biodegradable mPEG-PLGA-PLL nanoparticles loading Cy3-labelled PDGF-BB siRNA by UTMD to rat retina. $J$ Biosci 2017; 42: 299309.

[59] Kowalczuk L, Boudinet M, El Sanharawi M, Touchard E, Naud MC, 
Saïed A, et al. In vivo gene transfer into the ocular ciliary muscle mediated by ultrasound and microbubbles. Ultrasound Med Biol 2011; 37: 1814-1827.

[60] Fan CH, Cheng YH, Ting CY, Ho YJ, Hsu PH, Liu HL, et al. Ultrasound/magnetic targeting with SPIO-DOX-Microbubble Complex for image-guided drug delivery in brain tumors. Theranostics 2016; 6: 1542-1556.

[61] Kuo TT, Wang CH, Wang JY, Chiou HJ, Fan CH, Yeh CK. Concurrent osteosarcoma theranostic strategy using contrastenhanced ultrasound and drug-loaded bubbles. Pharmaceutics 2019; 11: 223 .

[62] Escoffre JM, Mannaris C, Geers B, Novell A, Lentacker I, Averkiou $\mathrm{M}$, et al. Doxorubicin liposome-loaded microbubbles for contrast imaging and ultrasound-triggered drug delivery. IEEE Trans Ultrason Ferroelectr Freq Control 2013; 60: 78-87.

[63] Nittayacharn P, Yuan HX, Hernandez C, Bielecki P, Zhou H, Exner AA. Enhancing tumor drug distribution with ultrasound-triggered nanobubbles. J Pharm Sci 2019; 108: 3091-3098.

[64] Ren ST, Liao YR, Kang XN, Li YP, Zhang H, Ai H, et al. The antitumor effect of a new docetaxel-loaded microbubble combined with low-frequency ultrasound in vitro: preparation and parameter analysis. Pharm Res 2013; 30: 1574-1585.

[65] Kang J, Wu X, Wang Z, Ran H, Xu C, Wu J, et al. Antitumor effect of docetaxel-loaded lipid microbubbles combined with ultrasoundtargeted microbubble activation on VX2 rabbit liver tumors. $J$ Ultrasound Med 2010; 29: 61-70.

[66] Yan F, Li L, Deng Z, Jin Q, Chen J, Yang W, et al. Paclitaxelliposome-microbubble complexes as ultrasound-triggered therapeutic drug delivery carriers. J Control Release 2013; 166: 246-255.

[67] Zhang J, Wang S, Deng Z, Li L, Tan G, Liu X, et al. Ultrasoundtriggered drug delivery for breast tumor therapy through iRGDtargeted paclitaxel-loaded liposome-microbubble complexes. $J$ Biomed Nanotechnol 2018; 14: 1384-1395.

[68] Ting CY, Fan CH, Liu HL, Huang CY, Hsieh HY, Yen TC, et al. Concurrent blood-brain barrier opening and local drug delivery using drug-carrying microbubbles and focused ultrasound for brain glioma treatment. Biomaterials 2012; 33: 704-712.

[69] Fan CH, Ting CY, Lin HJ, Wang CH, Liu HL, Yen TC, et al. SPIOconjugated, doxorubicin-loaded microbubbles for concurrent MRI and focused-ultrasound enhanced brain-tumor drug delivery. Biomaterials 2013; 34: 3706-3715.

[70] Gao S, Cheng X, Li J. Lipid nanobubbles as an ultrasound-triggered artesunate delivery system for imaging-guided, tumor-targeted chemotherapy. Onco Targets Ther 2019; 12: 1841-1850.

[71] Liang X, Xu Y, Gao C, Zhou Y, Zhang N, Dai Z. Ultrasound contrast agent microbubbles with ultrahigh loading capacity of camptothecin and floxuridine for enhancing tumor accumulation and combined chemotherapeutic efficacy. NPG Asia Mater 2018; 10: 761-774.

[72] Chen M, Liang X, Gao C, Zhao R, Zhang N, Wang S, et al. Ultrasound triggered conversion of porphyrin/camptothecinfluoroxyuridine triad microbubbles into nanoparticles overcomes multidrug resistance in colorectal cancer. ACS Nano 2018; 12: 7312 7326.

[73] Zhou Y, Gu H, Xu Y, Li F, Kuang S, Wang Z, et al. Targeted antiangiogenesis gene therapy using targeted cationic microbubbles conjugated with CD105 antibody compared with untargeted cationic and neutral microbubbles. Theranostics 2015; 5: 399-417.

[74] Fan CH, Chang EL, Ting CY, Lin YC, Liao EC, Huang CY, et al. Folate-conjugated gene-carrying microbubbles with focused ultrasound for concurrent blood-brain barrier opening and local gene delivery. Biomaterials 2016; 106: 46-57.

[75] Ji Y, Han Z, Shao L, Zhao Y. Evaluation of in vivo antitumor effects of low-frequency ultrasound-mediated miRNA-133a microbubble delivery in breast cancer. Cancer Med 2016; 5: 2534-2543.

[76] Cai W, Lv W, Feng Y, Yang H, Zhang Y, Yang G, et al. The therapeutic effect in gliomas of nanobubbles carrying siRNA combined with ultrasound-targeted destruction. Int J Nanomedicine 2018; 13: 6791-6807.

[77] Cavalli R, Soster M, Argenziano M. Nanobubbles: a promising efficient tool for therapeutic delivery. Ther Deliv 2016; 7: 117-138.

[78] Zha Z, Wang S, Zhang S, Qu E, Ke H, Wang J, et al. Targeted delivery of $\mathrm{CuS}$ nanoparticles through ultrasound image-guided microbubble destruction for efficient photothermal therapy. Nanoscale 2013; 5: 3216-3219.

[79] Zha Z, Wang J, Zhang S, Wang S, Qu E, Zhang Y, et al. Engineering of perfluorooctylbromide polypyrrole nano-/microcapsules for simultaneous contrast enhanced ultrasound imaging and photothermal treatment of cancer. Biomaterials 2014; 35: 287-293.

[80] Wang S, Dai Z, Ke H, Qu E, Qi X, Zhang K, et al. Contrast ultrasound-guided photothermal therapy using gold nanoshelled microcapsules in breast cancer. E J Radiol 2014; 83: 117-122.

[81] You Y, Liang X, Yin T, Chen M, Qiu C, Gao C, et al. Porphyringrafted lipid microbubbles for the enhanced efficacy of photodynamic therapy in prostate cancer through ultrasound-controlled in situ accumulation. Theranostics 2018; 8: 1665-1677.

[82] Zhao R, Liang X, Zhao B, Chen M, Liu R, Sun S, et al. Ultrasound assisted gene and photodynamic synergistic therapy with multifunctional FOXA1-siRNA loaded porphyrin microbubbles for enhancing therapeutic efficacy for breast cancer. Biomaterials 2018; 173: 58-70.

[83] Jang Y, Kim D, Lee H, Jang H, Park S, Kim GE, et al. Development of an ultrasound triggered nanomedicine-microbubble complex for chemo-photodynamic-gene therapy. Nanomedicine 2020: 102194.

[84] Xu Y, Liang X, Bhattarai P, Sun Y, Zhou Y, Wang S, et al. Enhancing therapeutic efficacy of combined cancer phototherapy by ultrasoundmediated in situ conversion of near-infrared cyanine/porphyrin microbubbles into nanoparticles. Adv Funct Mater 2017; 27 : 1704096.1.

[85] Postiglione I, Chiaviello A, Palumbo G. Enhancing photodynamyc therapy efficacy by combination therapy: dated, current and oncoming strategies. Cancers 2011; 3: 2597-2629. 